\title{
Structural Health Monitoring Based on Combined Structural Global and Local Frequencies
}

\author{
Jilin Hou, ${ }^{1}$ Lukasz Jankowski, ${ }^{2}$ and Jinping $O \mathrm{u}^{1,3}$ \\ ${ }^{1}$ School of Civil Engineering, Dalian University of Technology, Dalian 116024, China \\ ${ }^{2}$ Institute of Fundamental Technological Research, Polish Academy of Sciences, 02-106 Warsaw, Poland \\ ${ }^{3}$ School of Civil Engineering, Harbin Institute of Technology, Harbin 150090, China \\ Correspondence should be addressed to Jilin Hou; hou.jilin@hotmail.com
}

Received 9 August 2013; Accepted 5 December 2013; Published 9 January 2014

Academic Editor: Yuri Petryna

Copyright (C) 2014 Jilin Hou et al. This is an open access article distributed under the Creative Commons Attribution License, which permits unrestricted use, distribution, and reproduction in any medium, provided the original work is properly cited.

\begin{abstract}
This paper presents a parameter estimation method for Structural Health Monitoring based on the combined measured structural global frequencies and structural local frequencies. First, the global test is experimented to obtain the low order modes which can reflect the global information of the structure. Secondly, the mass is added on the member of structure to increase the local dynamic characteristic and to make the member have local primary frequency, which belongs to structural local frequency and is sensitive to local parameters. Then the parameters of the structure can be optimized accurately using the combined structural global frequencies and structural local frequencies. The effectiveness and accuracy of the proposed method are verified by the experiment of a space truss.
\end{abstract}

\section{Introduction}

Structural Health Monitoring (SHM) [1] is a hot research in civil engineering. Structural parameter estimation, such as model updating and damage identification $[2,3]$, is an important theoretical base for providing reliable model, structural damage detection, forecasting, and its safety assessment.

In SHM, many researches have investigated parameter estimation like model updating, damage detection and localization, and so forth. The structural parameters are usually optimized using natural frequency and mode shape [4], flexibility matrix [5], responses in time-domain or frequencydomain [6], and so forth. Among the dynamic information, natural frequency reflects the most basic dynamic performance of the structure, and it can be identified easily, accurately, robustly, and reliably. So the methods based on mode are widely used for parameters estimation. In 2000, Yun and Bahng [7] monitor the local stiffness modifications using natural frequencies and mode shapes. In 2007, Jaishi and Ren [8] update the parameters of the finite element model based on eigenvalue and strain energy residuals. In 2011, An and Ou [9] detect local damages of a truss structure using local mode.
Usually, the identified low order natural frequencies are not enough for estimating the structural parameters in civil engineering. Aiming at solving this problem, adding mass on structure is an effective way to increase dynamic information. In 1992, Nalitolela et al. [10] add mass or stiffness on the structure for model updating, where the mode of the perturbed structures is used. In 1993, Nalitolela et al. [11] further improve their method by adding imaged stiffness on the beam. In 2001, Cha and Pillis [12] use the orthogonal conditions of the system's eigenvalue problem for identifying damage by adding known masses on the structure. In 2011 Dinh et al. [13] use the state-space transformation of the system's eigenvalue problem to improve the method.

However, with structures in civil engineering being more and more huge and complexity, the accurate parameter estimation of structure has becoming more and more difficult, which requires the proper dynamic measurements, as well as the efficient exploitation of the measurements. Therefore, this paper combined both the structural global frequencies and structural local frequencies for parameter estimation, and the method of adding mass is employed to make the local structure have Local Primary Frequency (LPF) [14], 


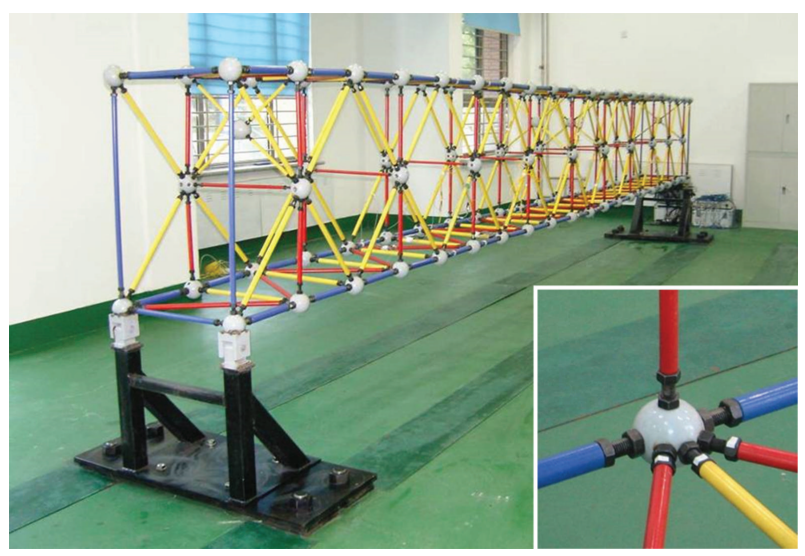

FIgURE 1: A truss experimental model.

which not only increases the useful measured information but also improves its sensitivity to the local parameters. The experiment of a space truss is performed for the verification of the proposed method.

\section{SHM by Combined Global and Local Mode}

Structures in civil engineering are large scale, complex, and insensitive to the damage, and hence the accurate estimation of their structural parameters requires the utilization of a great quantity of their dynamic information. Modal information including frequency and vibration mode is most commonly used in Structural Health Monitoring (SHM). In this paper, structural global frequencies are used together with local frequencies to estimate physical parameters of the structures.

2.1. Structural Global Frequency. Generally, structural global modes reflect the overall dynamic characteristics of the structure, and thereby they have certain relation with physical quantities related to the integrity, such as the elastic modulus and the density. For global modes, usually only low-order modes can be obtained in practice from the global measurements. However low-order modes are usually insensitive to the variation of structural local parameters. For instance, the first-order mode of a large-scale structure generally changes little due to the cause that only one structural element is damaged.

In civil engineering area, the modes of the large-scale structures are often identified by the methods like Eigensystem Realization Algorithm (ERA), Stochastic Subspace Identification method (SSI), and so on, using the free responses or random responses. Then the parameter estimation is performed based on the identified modes. At present, these methods based on identified modes [1-4, 8,9] have been intensively investigated and so they are not introduced in detail here.

2.2. Structural Local Frequency. As well known, structural local frequency is a type of mode that can reflect local dynamic characteristics of the local structure and is sensitive to the variation of local parameters. Generally speaking, structural local frequency refers to a high-order structural mode. Compared with the obtained structural global frequency, structural local mode mainly displays local dynamic characteristics of the structure. Thus, using local structural frequency, the structural local parameters can be evaluated more accurately.

First, Local Primary Frequency (LPF) is illustrated in the following subsection, which refers to one natural frequency corresponding to the vibration of the local structure under certain excitations.

2.2.1. Local Primary Frequency (LPF). The acceleration frequency response of the structure $H(\omega)$ can be expressed as

$$
H(\omega)=\sum_{r=1}^{n} \frac{(j \omega)^{2} C \varphi_{r} \varphi_{r}^{T} B}{\omega_{r}^{2}-\omega^{2}+j 2 \zeta_{r} \omega_{r} \omega},
$$

where $j$ is imaginary unit, $\zeta_{r}$ is the $r$ th damping ratio, $\omega_{r}$ is the $r$ th natural frequency, $\varphi_{r}$ is the $r$ th normalized mode shape, $B$ is load position matrix, and $C$ is observation matrix.

Substitute $\omega=\omega_{r}$ into (1); then the corresponding frequency response $H\left(\omega_{r}\right)$ has the approximate expression as follows:

$$
H\left(\omega_{r}\right) \approx \frac{j C \varphi_{r} \varphi_{r}^{T} B}{2 \zeta_{r}} .
$$

It can be seen from (2) that, apart from the modal parameters of the structure, the measured response of the structure mainly depends on the excitation and the sensor positions. Therefore, to obtain the structural local frequencies of the structure, the sensors and excitation are required to be placed locally.

Usually, more than one natural frequency is likely to be excited even under local excitation. Among these natural frequencies, there are not only structural local frequencies, but the frequencies which represent the structural global information are also included. In practice, it is difficult to accurately separate high-sensitivity local frequencies from these frequencies. In this way, Local Primary Frequency (LPF) is defined: under local excitation, if the structure mainly vibrates in local positions with one main natural frequency, then the natural frequency is defined as LPF. The LPF is highly sensitive to the corresponding local parameters, which is a crucial factor in the proposed method of the structural parameter estimation. Obtainment of LPF is analyzed in Section 2.2.3, and before that the related sensitivity analysis is discussed.

2.2.2. Sensitivity Analysis. Let a linear structure have $n$ degrees of freedom (Dofs), and denote by $K_{0}$ and $M_{0}$, respectively, the stiffness matrix and mass matrix of the theoretical FEM model structure. Assume the structure has $m$ parameters to be estimated, which are only related to the stiffness. The parameter modification coefficients are denoted by $\mu_{1}, \mu_{2}, \ldots, \mu_{n}$, which are, respectively, the ratio of the actual modified stiffness matrix $K_{i}^{d}$ to the stiffness matrix 


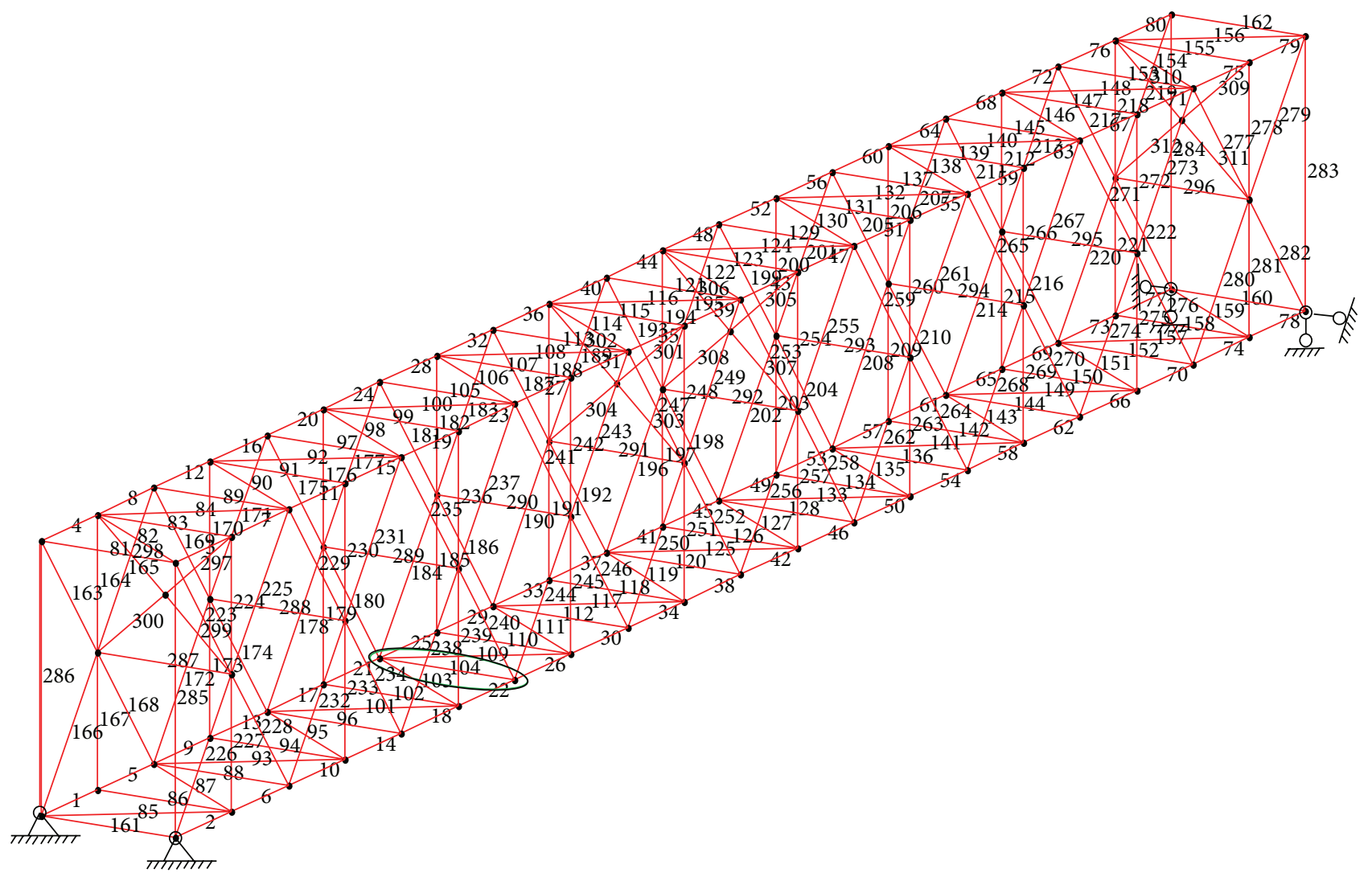

Figure 2: The element numbers of truss model.

of theoretical FEM model $K_{i}$. The stiffness matrix of real structure $K$ is expressed as

$$
K^{d}=\sum \mu_{i} K_{i}, \quad K_{i}=N_{i}^{T} K_{i, s} N_{i},
$$

where $K_{i}$ is the $i$ th extended substructure stiffness matrix of theoretical FEM model, $K_{i, s}$ is the corresponding substructure matrix in local coordinate system, $N_{i}$ is the localization matrix linking the global Dofs to the $i$ th local substructural Dofs.

Via the relation of the natural frequency to the substructure stiffness matrix, the sensitivity of the $r$ th natural frequency $\omega_{r}$ to the $i$ th parameter modification coefficient $\mu_{i}$ can be stated as

$$
\frac{\partial \omega_{r}}{\partial \mu_{i}}=\frac{\varphi_{r}^{T} K_{i} \varphi_{r}}{2 \omega_{r}}=\frac{\varphi_{r, i}^{T} K_{i, s} \varphi_{r, i}}{2 \omega_{r}},
$$

where $\varphi_{r, i}=N_{i} \varphi_{r}$ is the $r$ th mass-normalized mode shape related to the $i$ th parameter. Then, the sum of the relative sensitivity of all the parameters can be computed as follows [15]

$$
\sum_{i} \frac{1}{\omega_{r}} \frac{\partial \omega_{r}}{\partial \mu_{i}}=\sum_{i} \frac{\varphi_{r}^{T} K_{i} \varphi_{r}}{2 \omega_{r}^{2}}=\frac{1}{2}
$$

2.2.3. Adding Masses and LPF. Generally, the response of local substructure doesn't have LPF. Hence, masses are added artificially on local structure to cause the local structure have a LPF and make it mainly vibrate at the LPF. As a part of the local structure, the added mass can increase the vibration energy of the local structure and restrain the vibration energy dispersion from local structure to global structure. As a result, the local structure may vibrate with one natural frequency, that is, the defined Local Primary Frequency.

Comparing (2) and (4), it can be seen that the expression of the frequency response is similar on the structure to the expression of the relative sensitivity. Therefore, it can be inferred that there is a certain correlation between the amplitude of the frequency response and the sensitivity: the larger the amplitude is, the higher the corresponding sensitivity may be. That is because, under local excitation, the structural frequency response shows the maximum amplitude at the position of the caused LPF. Moreover, this indicates that LPF is more sensitive to local parameters than other natural frequencies.

In addition, as shown in (5), the sum of the relative sensitivity of a natural frequency to all parameters is a constant value of 0.5 . Hence, if the relative sensitivity of LPF according to the concerned local parameter is higher, then the LPF will have lower sensitivity to other parameters. Therefore, the accuracy of the parameter estimation can be improved using LPF to optimization.

2.3. The Objective Function. The modification of the concerned parameters is taken as the optimization variables, 


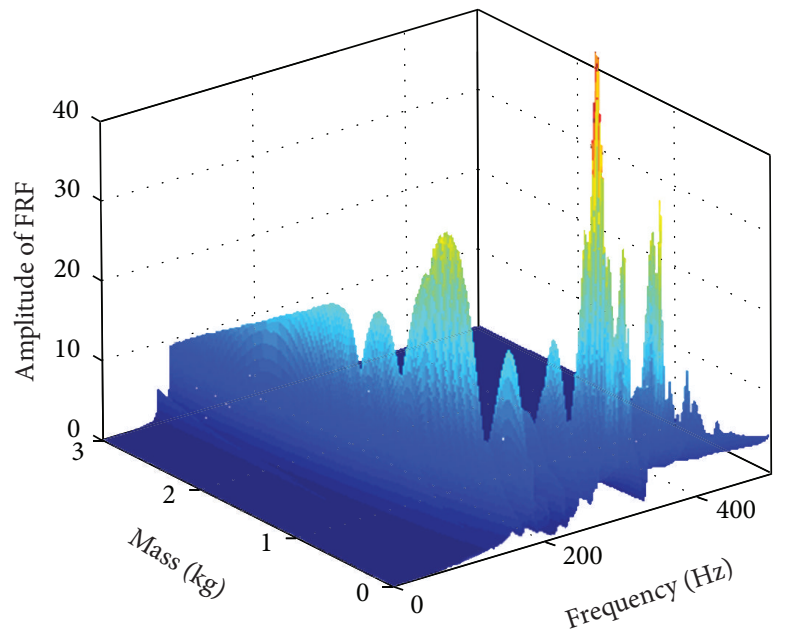

(a) Frequency responses

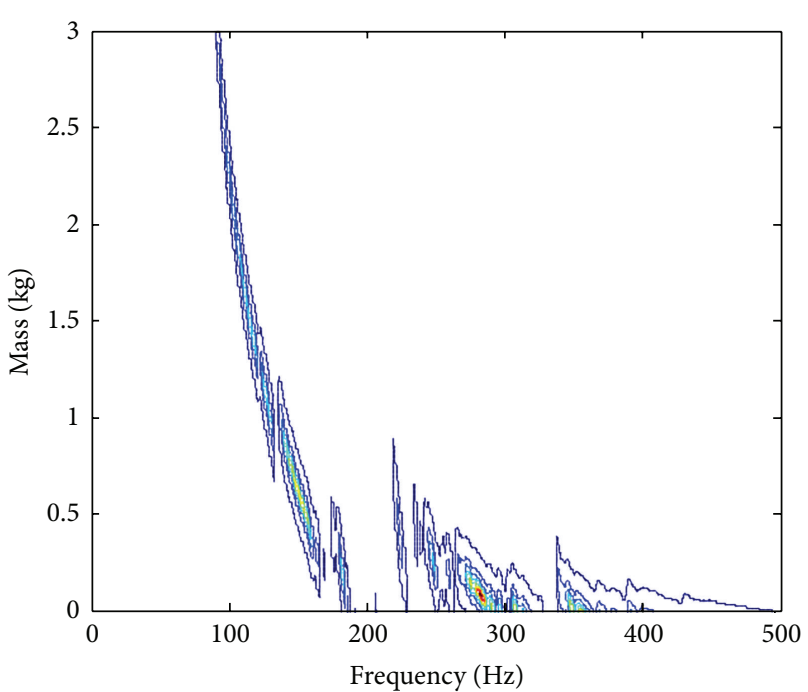

(b) Contour line

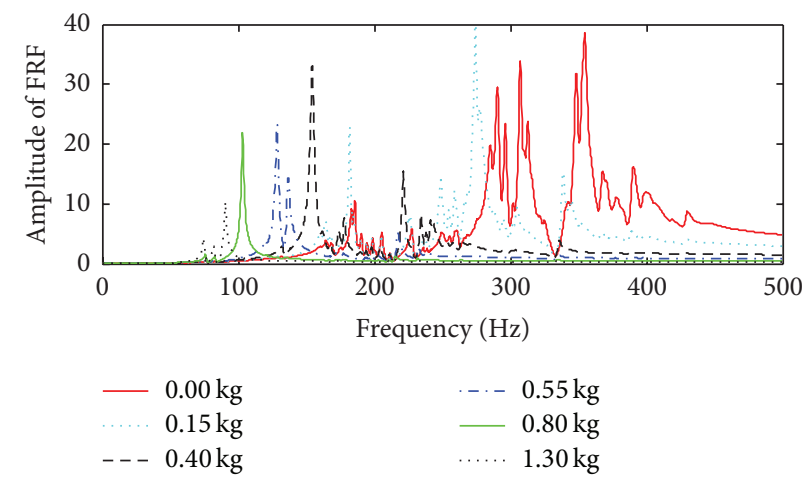

(c) Frequency responses in 6 groups of different added masses

Figure 3: The out-plane frequency responses of the truss after adding masses.

and the objective function $f(\mu)$ is built via combing the information of both global structural frequencies and LPF:

$$
f(\mu)=\sum_{i}\left\|\frac{\omega_{g, i}(\mu)-\bar{\omega}_{g, i}}{\bar{\omega}_{g, i}}\right\|+\sum_{j}\left\|\frac{\omega_{s, j}(\mu)-\bar{\omega}_{s, j}}{\bar{\omega}_{s, j}}\right\|,
$$

where $\mu$ is the variable to be identified; $\bar{\omega}_{g, i}$ is the identified $i$ th low-order natural frequency of the global structure, while $\omega_{g, i}(\mu)$ is the corresponding frequency of the theoretical FEM model; $\bar{\omega}_{s, j}$ is the measured LPF of the $j$ th local parameter, while $\omega_{s, j}(\mu)$ is the corresponding LPF of the theoretical FEM model.

\section{Truss Experiment}

An experiment of a space truss model is performed here to introduce and test the proposed method by combining the global and local frequency.

3.1. Truss Model. A supported steel 20-span space truss is shown in Figure 1. It is $8 \mathrm{~m}$ long with the height of $0.9 \mathrm{~m}$, width of $0.56 \mathrm{~m}$, and consists of 312 members and 108 nodes.
TABLE 1: Three types of members in truss structure.

\begin{tabular}{lccc}
\hline Types & Member A & Member B & Member C \\
\hline Number & 80 & 198 & 34 \\
\hline Tubes & & & \\
$\quad$ Outside diameter $(\mathrm{mm})$ & 30 & 20 & 14 \\
$\quad$ Thick $(\mathrm{mm})$ & 8 & 3 & 3 \\
\hline Bolt & & & \\
$\quad$ Types & M20 & M16 & M10 \\
$\quad$ Length $(\mathrm{mm})$ & 100 & 90 & 60 \\
\hline
\end{tabular}

Young's modulus is $2.06 \mathrm{Gpa}$, and the density is $7850 \mathrm{Kg} / \mathrm{m}^{3}$. The members, steel tubes, are connected by bolted spherical joints (bottom right in Figure 1). The members are classified into three types according to their radius, which are listed in Table 1 .

The theoretical Finite Element (FE) model is built, where each member is defined as an element. Then the FE model has 312 elements with 108 nodes and 638 degrees of freedom. Figure 2 shows the element numbers of the truss model. The element cross-sections and initial moments are calculated 


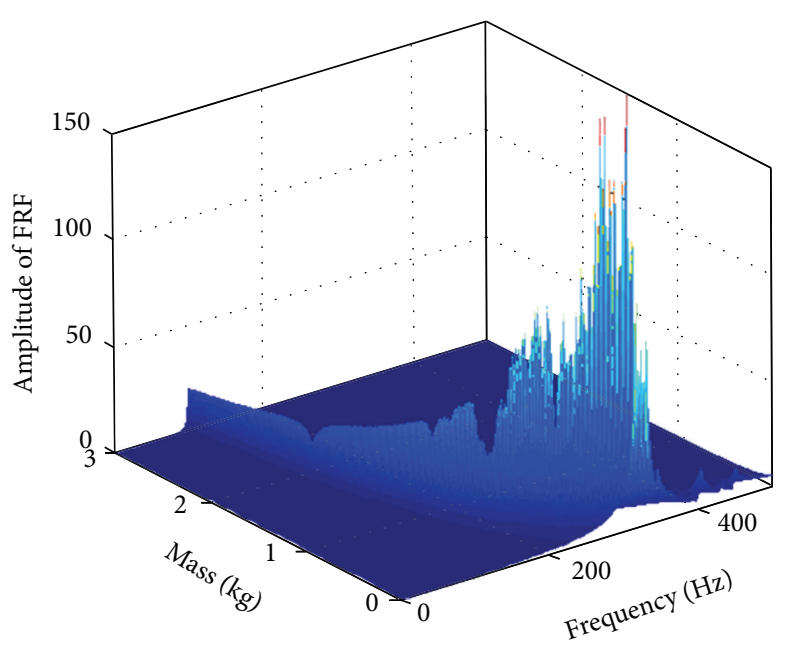

(a) Frequency responses

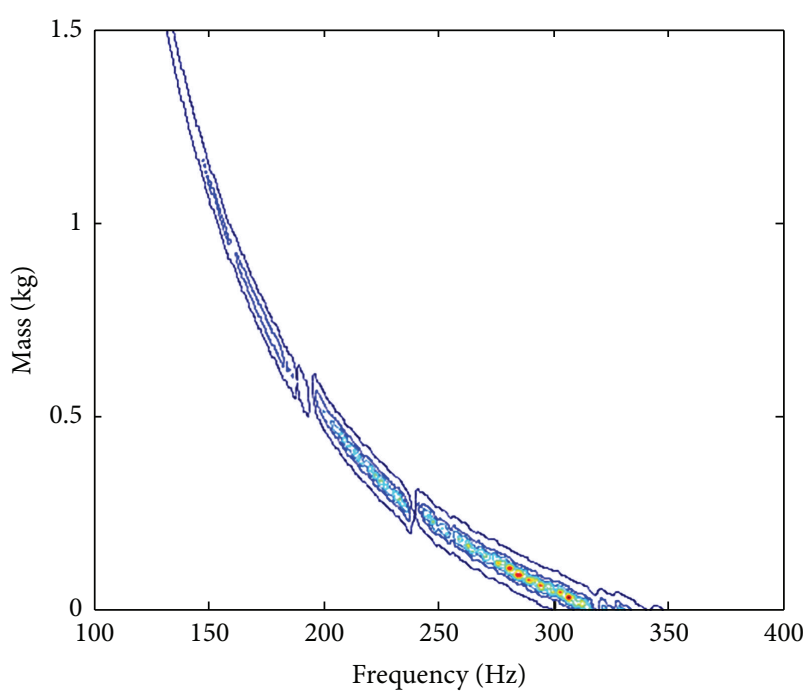

(b) Contour line

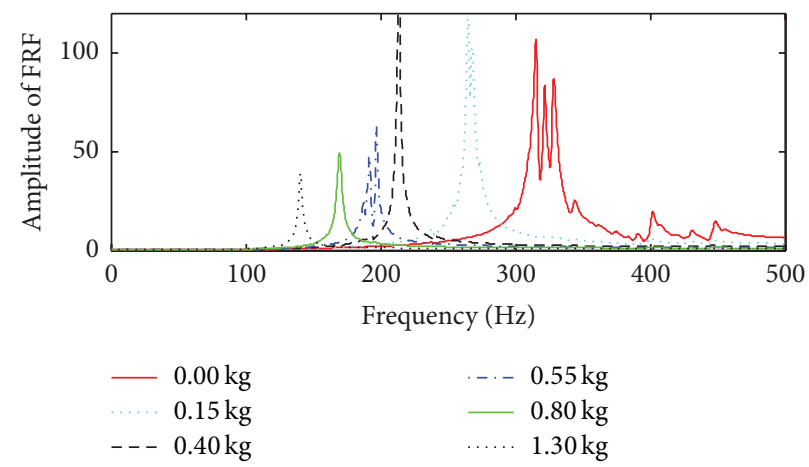

(c) Frequency responses in 6 groups of different added masses

FIGURE 4: The in-plane frequency response after adding masses.

from the geometric dimensioning according to the steel tube. The nodes are rigid. The weight of the spherical joints and bolts is considered by certain mass points located on the nodes. Each mass point has the value of $2.83 \mathrm{~kg}$.

3.2. Numerical Analysis of LPF. In order to investigate the influence of the added mass on LPF, the bottom element number 104 of the FE model is taken as an example, marked by a black circle in Figure 2, and the analysis is performed numerically. The mass is added in the middle of member number 104 and the impulse excitation is also applied in the middle of the member. After the mass addition, the in-plane frequency response of the member and the outplane frequency response are compared, respectively (here, the in-plane refers to the one corresponding to the outer contour of the truss). Figures 3 and 4, respectively, show the corresponding in-plane and out-plane frequency responses in 6 groups of different added masses (mass $=0.00,0.15$, $0.40,0.55,0.80$, and $1.30 \mathrm{~kg}$ ). Figures 3 and 4 both provide similar phenomenon. The increase in mass causes the peaks of the frequency response change from multifrequencies into a single frequency (the caused LPF). But with the increase in mass, the value of LPF is reduced. When the value of LPF reduction reaches a certain degree, the frequency response will appear multipeaks. Then if the added mass value is continuing to increase, the peaks of frequency response return to a single peak again. The comparison between Figures 3 and 4 indicates that, under the same additional mass, the in-plane LPF is larger than the out-plane LPF. The member section is a circle and member stiffness is identical in all directions, and further the whole truss owns larger in-plane stiffness at boundary of the member; therefore, with the same additional mass value, the in-plane local characteristics of the member are more significant. Hence, the in-plane response of the member is used for the estimation in the test of experiment.

For each in-plane frequency response shown in Figure 4(c), the sensitivity analysis of the natural frequencies corresponding to the peaks is computed, respectively, which are shown in Figure 5. When no mass is added, the frequency response in Figure 3(c) shows three peaks at the respective frequency of $315.4 \mathrm{~Hz}, 321.9 \mathrm{~Hz}$, and $328.0 \mathrm{~Hz}$. The relative sensitivities of the corresponding three natural frequencies are illustrated in Figure 5(a): around 0.08, 0.06, and 0.05, respectively. It shows that the three relative sensitivities are far larger than the sensitivities of the rest 311 members. 

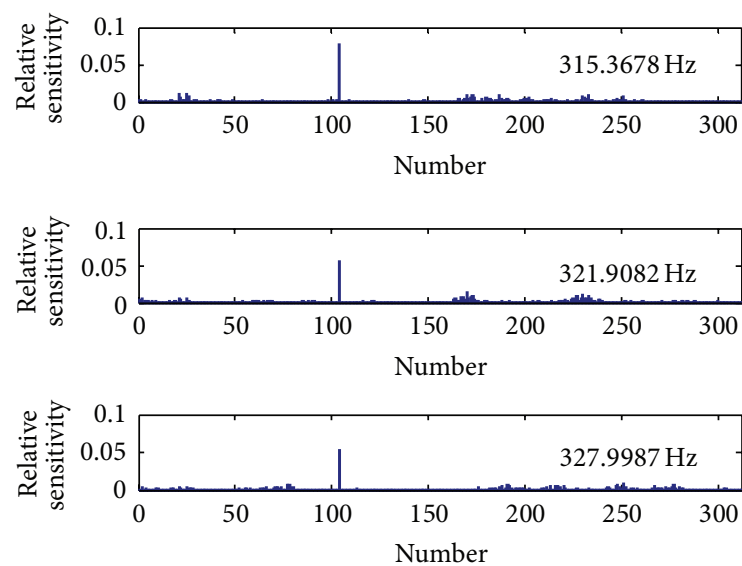

(a) $m=0.00 \mathrm{~kg}$

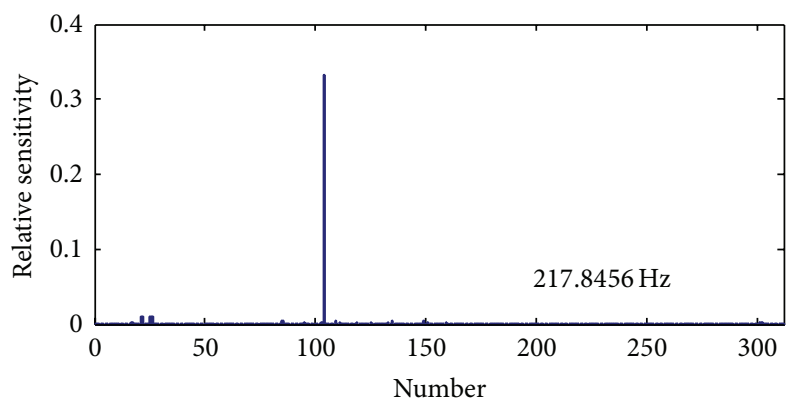

(c) $m=0.40 \mathrm{~kg}$

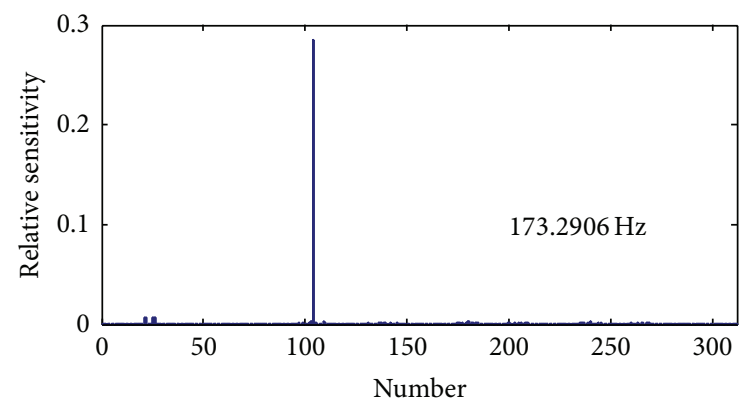

(e) $m=0.80 \mathrm{~kg}$
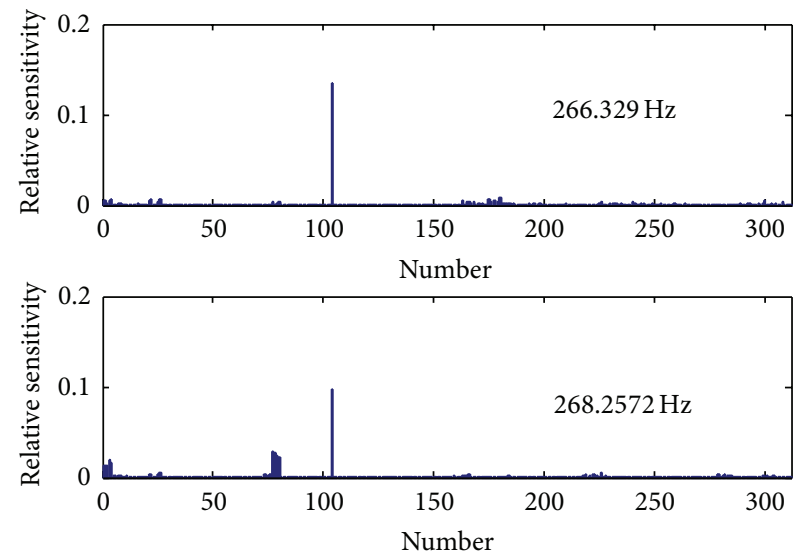

(b) $m=0.15 \mathrm{~kg}$
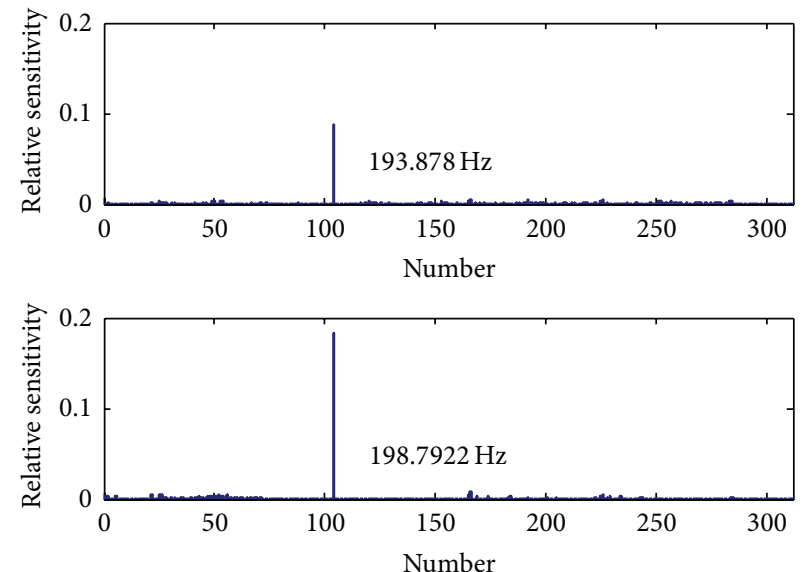

(d) $m=0.55 \mathrm{~kg}$

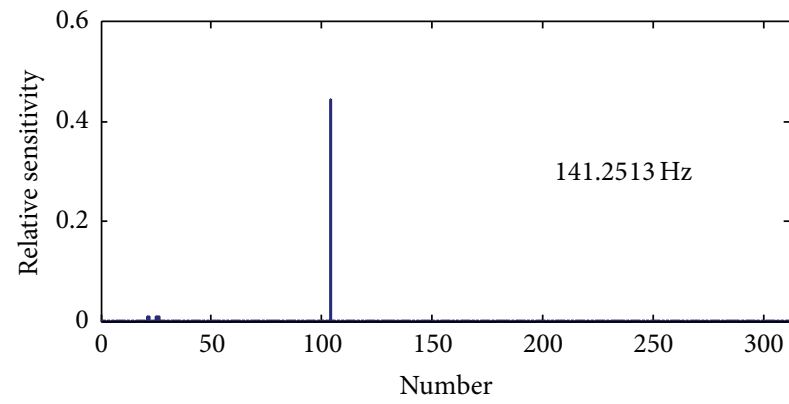

(f) $m=1.30 \mathrm{~kg}$

FIgURE 5: The relative sensitivities of the excited frequencies in 6 cases of adding mass.

Equation (5) shows that the sum of all the relative sensitivities is 0.5 , so it can be inferred that the influence of the number 104 member on the three order frequencies only accounts for $0.08 / 0.5=16 \%, 0.06 / 0.5=12 \%$, and $0.05 / 0.5=10 \%$, respectively. When the added mass is $0.15 \mathrm{~kg}$, the two natural frequencies corresponding to the peaks of the frequency response have the relative sensitivities of 0.13 and 0.11 , respectively, as exhibited in Figure 5(b). When the added mass is $0.40 \mathrm{~kg}$, there is only one peak and the relative sensitivity of the corresponding natural frequency is 0.33 , as shown in Figure 5(c). Apparently, the more the peaks of the excited response, the lower the sensitivity of the corresponding natural frequency. When the added mass is $0.55 \mathrm{~kg}$, due to the change of modal order, the frequency response shows two peaks and the relative sensitivities of the corresponding frequencies are decreased to 0.1 and 0.18 , respectively, as shown in Figure 5(d). As the mass keeps on increasing, the frequency response changes from multiple peaks to a single peak and the corresponding sensitivities show a significant increase. When the mass is 0.8 and $1.3 \mathrm{~kg}$, the sensitivities of the corresponding frequencies are, respectively, 0.28 and 0.43 , as displayed in Figures 5(e) and 5(f). 


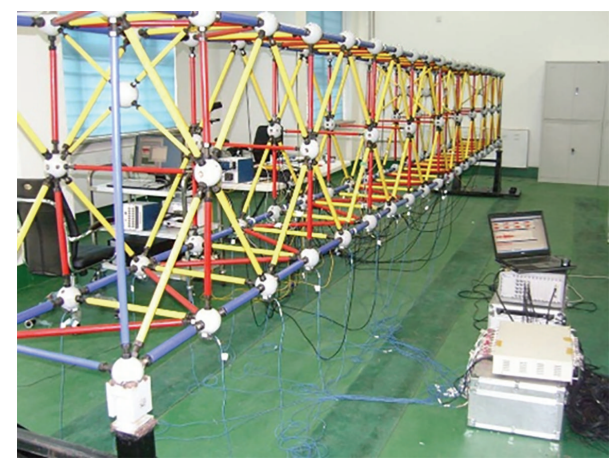

FIgure 6: Accelerations placement.
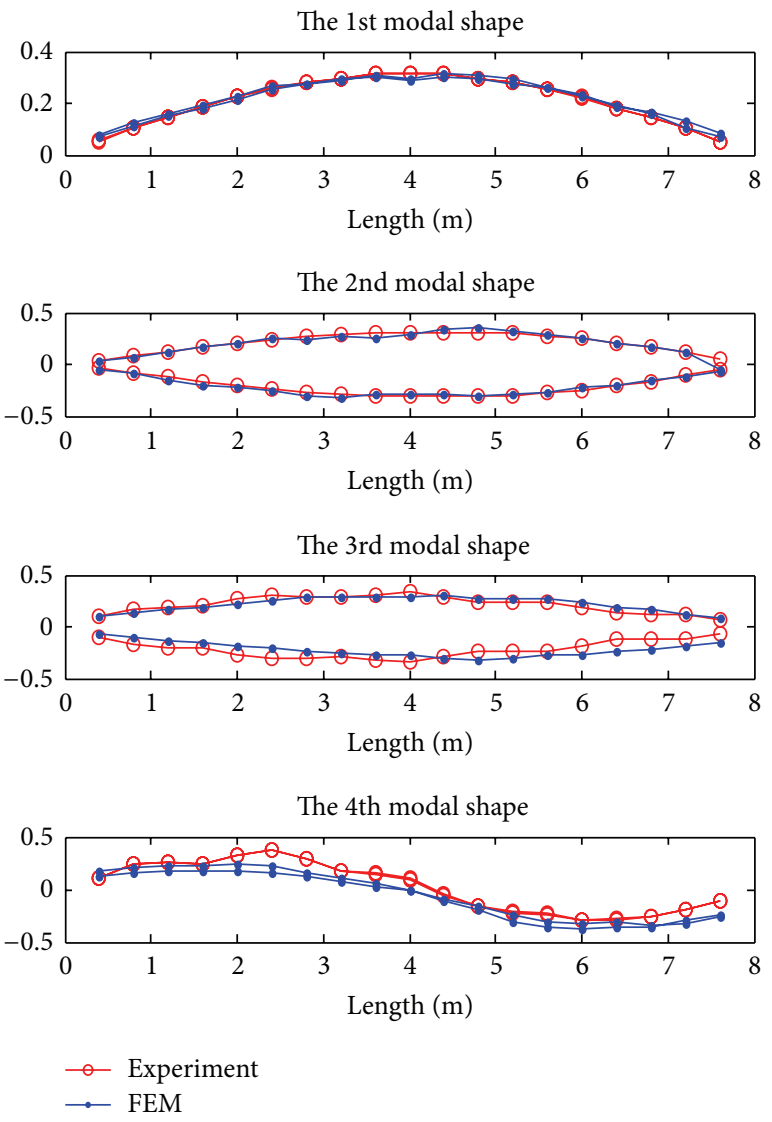

FIGURE 7: The comparison of modes of global structure.

The above numerical analysis among the relation of the adding mass, LPF, and the sensitivity provides the evidence for the following experiment performance.

\subsection{Model Updating of the Space Truss Experimentally}

3.3.1. Modal Test of the Global Truss. Accelerometers are located on the bottom nodes of the truss, shown in Figure 6. Ambient excitation is applied on the structure, and the modal shapes and frequencies are identified via the caused random responses, shown in Figure 7 and Table 2. Figure 7 and Table 2, respectively, compare the modal shapes and
TABLE 2: The errors of lower order natural frequencies of global structure.

\begin{tabular}{lccc}
\hline Order & Experimental & Theoretical & Error \\
\hline 1 & 18.4 & 23.27 & $26.47 \%$ \\
2 & 27.6 & 34.41 & $24.67 \%$ \\
3 & 36.8 & 52.58 & $42.89 \%$ \\
4 & 53.3 & 76.97 & $44.41 \%$ \\
\hline
\end{tabular}

frequencies which are obtained from the experimental model and FEM model. Table 2 shows that the errors between them are rather obvious, around $30 \%$, which indicates that the theoretical FEM model is quite coarse.

3.3.2. Local Dynamic Test. Since member B in Table 1 includes most of the members, the local dynamic test is performed on the group of member B, which are further classified into four kinds according to their lengths and positions (Figure 8). In order to increase the local dynamic characteristic, masses are added in the middle of the members. Due to that the weight of one accelerometer and its connector is $340 \mathrm{~g}$, so in the experiment the accelerometer also can play the role of additional mass, and the value of added mass is adjusted by the number of the located accelerometers. For long member, its local stiffness is relatively small, and one additional mass is enough; that is, one accelerometer is located. For short member, its local stiffness is big, and more masses (accelerometers) are needed. The location of the accelerometers or additional masses is shown in Figure 9.

Members-IV is first taken as an example to illustrate and experimentally verify that local characteristic can be increased via adding mass on the corresponding member. Three cases are discussed; that is, two, three, and four masses (accelerometers) are, respectively, added on middle of the number 251 member. In each case, a hammer excitation is applied on the middle of the member, and the corresponding responses of accelerometers are measured; then the spectral analysis is performed. The obtained power spectra in the three cases are shown in Figure 10. The bigger the additional mass value is, the more obvious the caused local characteristic will be. In the cases of two or three additional masses, the power spectra of the global structure have multiple peaks. So it is hard to judge which natural frequencies belong to the local structure and which frequencies reflect the characteristic of the global structure; that is to say, the local characteristic is not obvious. While in the case of four additional masses the power spectra only have one peak, it shows that the energy of the local excitation is mostly absorbed by the additional masses. In this case, the excited vibration mainly reflects the distortion of the local member, so the frequency corresponding to the peak in the power spectra is the LPF of the local member.

In order to compare the measured LPF and that of the theoretical model, 58 members are picked up to test, which are, respectively, 14 members from Members-I, 16 members from Members-II, 20 members from Members-III, and 8 members from Members-IV. The accelerometers (masses) are located on these members, respectively, according to Figure 9, 


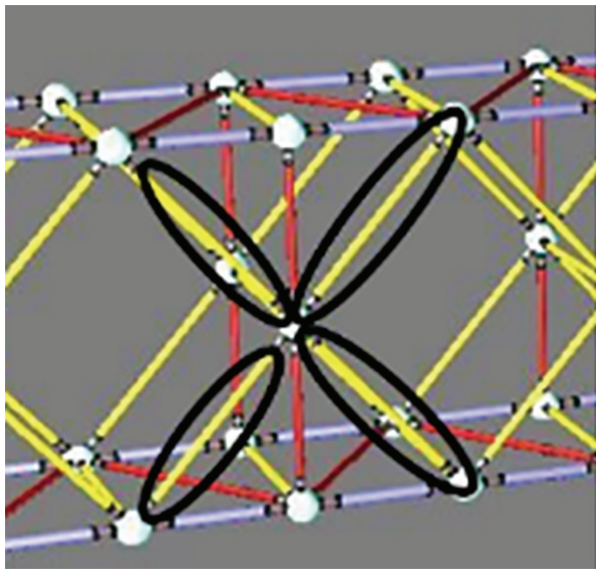

(a) Members-I

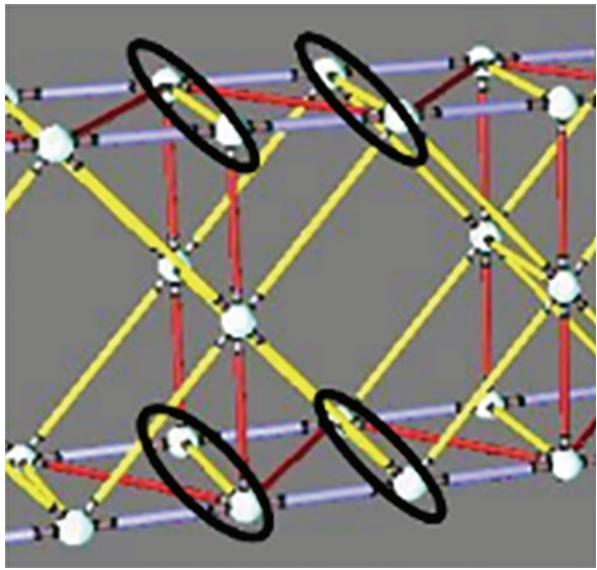

(c) Members-III

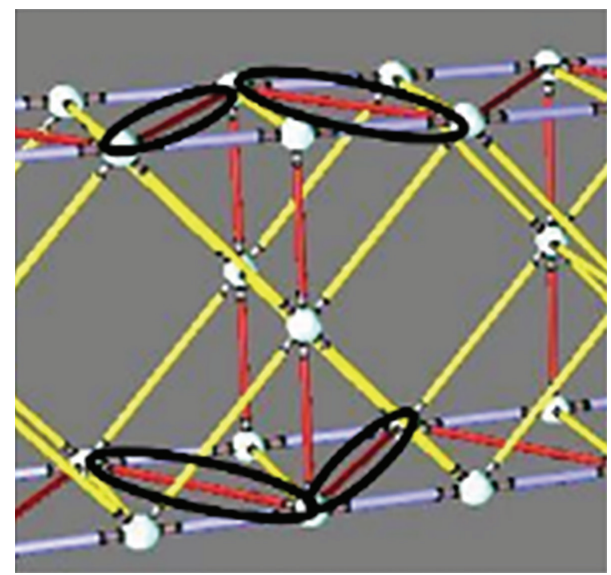

(b) Members-II

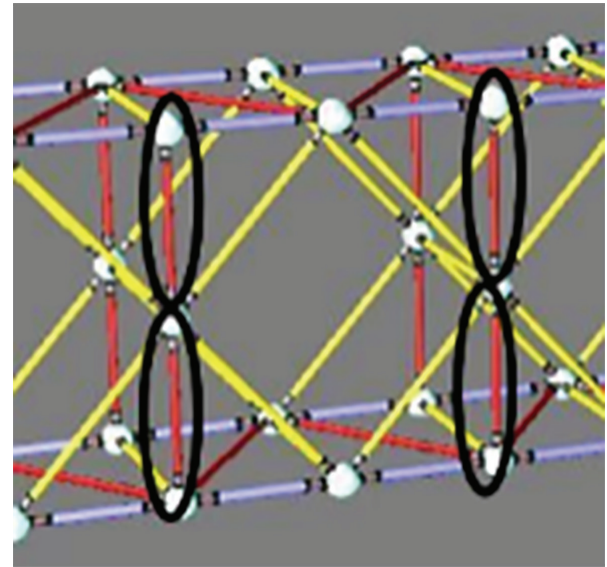

(d) Members-IV

Figure 8: Four kinds of elements.

TABLE 3: The errors of local primary frequencies of 4 kinds of elements.

\begin{tabular}{lccc}
\hline Types & Identified $(\mathrm{Hz})$ & Theoretical $(\mathrm{Hz})$ & Error \\
\hline Members-I & 138.67 & 151.24 & $9.06 \%$ \\
Members-II & 140.63 & 155.57 & $10.62 \%$ \\
Members-III & 166.02 & 176.28 & $6.18 \%$ \\
Members-IV & 164.06 & 186.52 & $13.69 \%$ \\
\hline
\end{tabular}

and the respective hammer excitation is applied in the middle. The identified LPFs are shown in Figure 11. Take Members-I as an example; the 14 members show nearly identical LPF; see Figure 11(a). The main reason is summarized as follows: these members have the same material and geometric parameters in the global structure. For these members, each member and its surrounding nodes are connected in a similar way; also, the connection way between one member and other members is similar. Restraining stiffness of the global structure on the two ends of the member is also very approximate to each other and the local excitation was in the same position and direction for the 14 members. Furthermore, the local characteristics of the members are amplified by the added masses. Therefore, with all the similar or identical conditions mentioned above, the LPFs of the members are similar to each other, although the members are at different positions in the global structure.

Table 3 compares the measured LPF and that of the theoretical FE model, where one member is chosen from each kind of members, respectively. It shows that the identified value from the experiment has obvious deviation from the theoretical value, which indicates that the theoretical model has significant error compared to the actual structure.

3.3.3. The Selection of Updating Parameters. In practice, actual Young's modulus of the steel, physical dimension, and the density have little error from the theoretical values. Then in this truss model, the factor of the errors of FEM model is mainly due to the node model, and so the parameters of node model are estimated in the model updating.

First the fine model of the bolted spherical joint, bolt, and the steel pipe are built considering their actual way of connection, shown in Figure 12. The local reinforcement of the spherical joint $(r=4 \mathrm{~cm})$ to the ends of the tube is considered by a reinforcement parameter $k$ which is the ratio of the spherical joint stiffness to the pipe stiffness, 


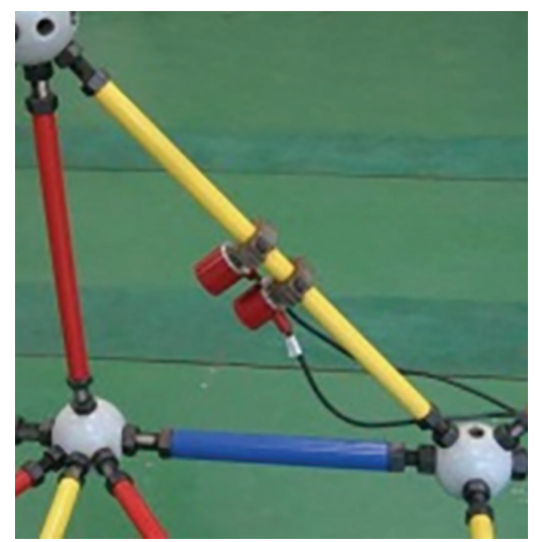

(a) Members-I

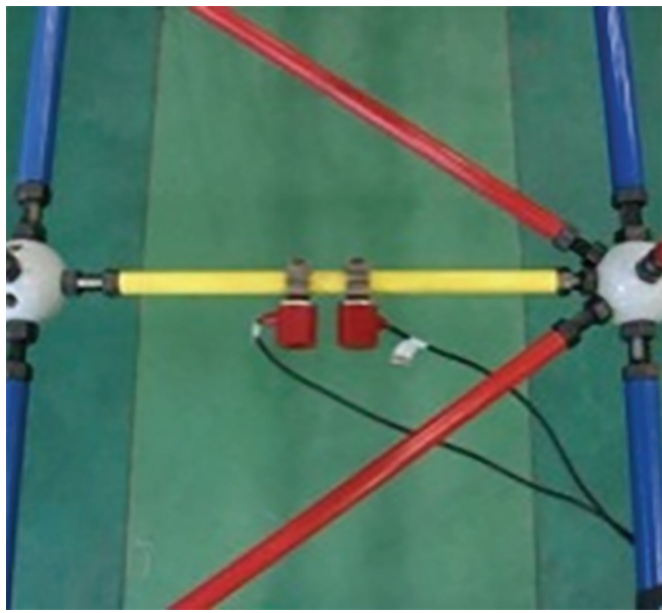

(c) Members-III

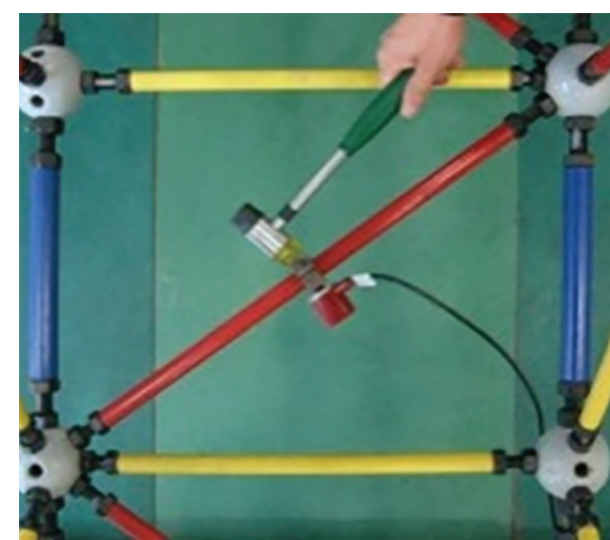

(b) Members-II

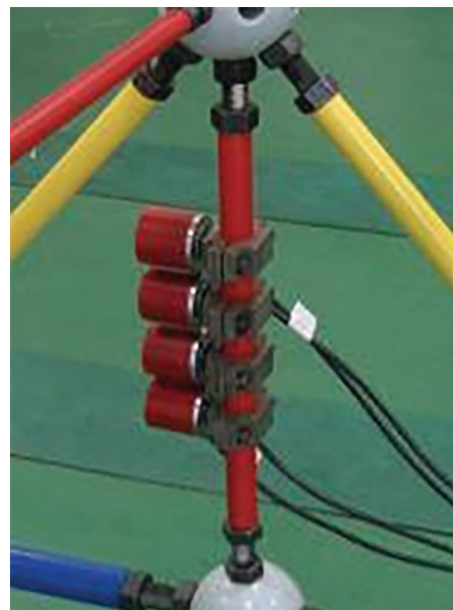

(d) Members-IV

FIgURE 9: The placements of additional masses.

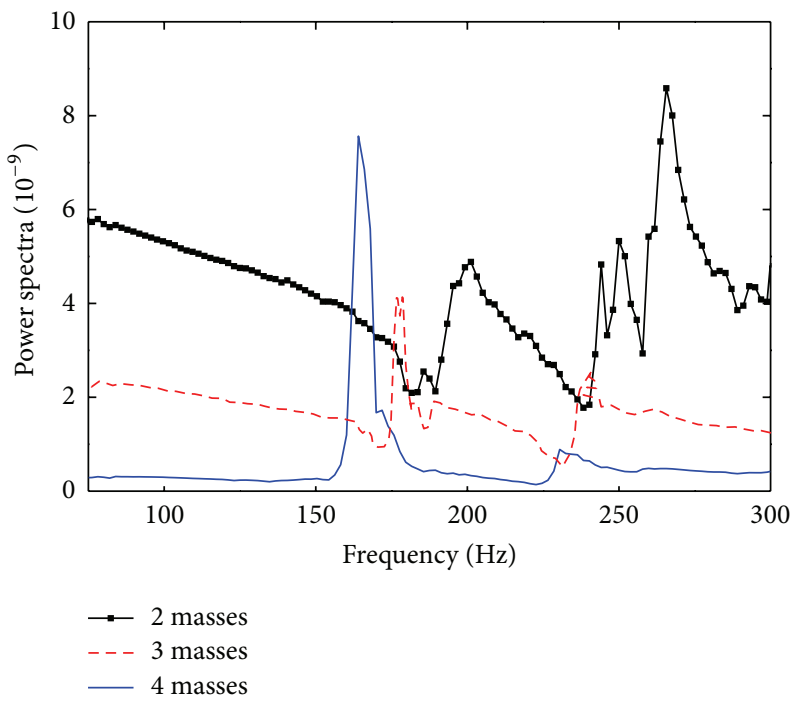

FIgURE 10: The power spectra. 


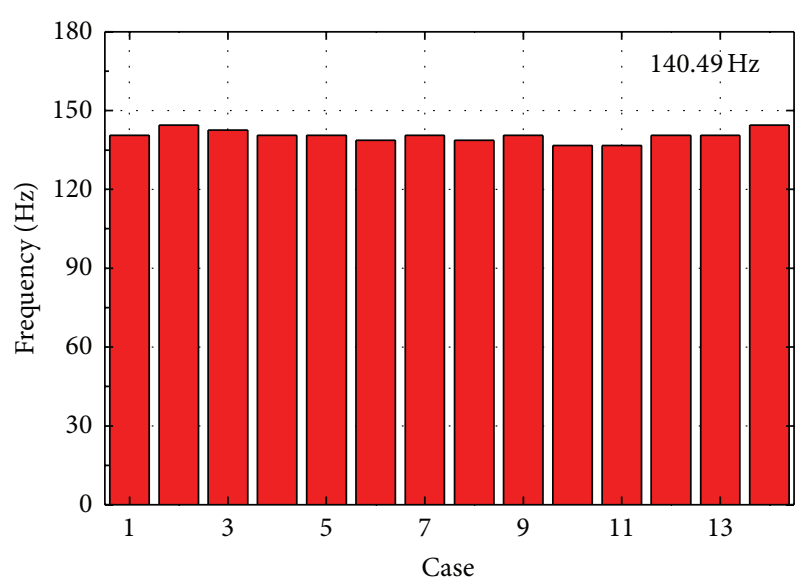

(a) Members-I

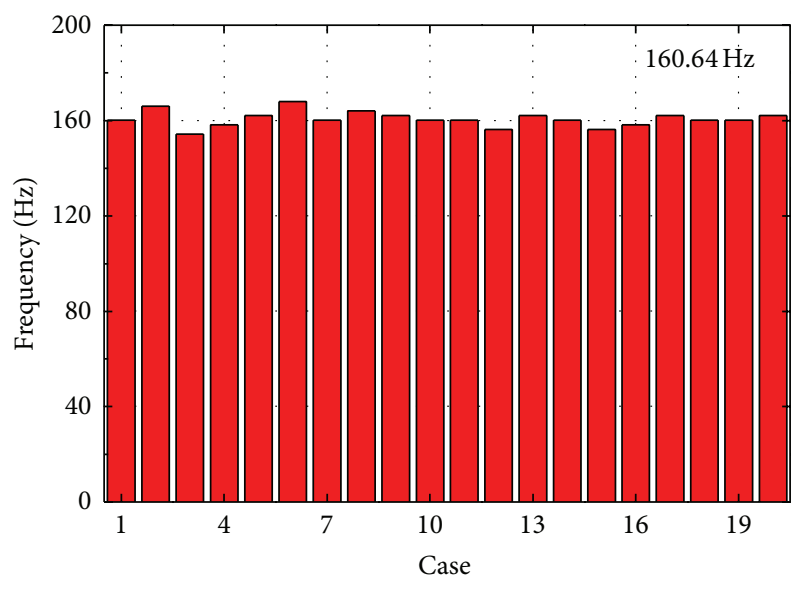

(c) Members-III

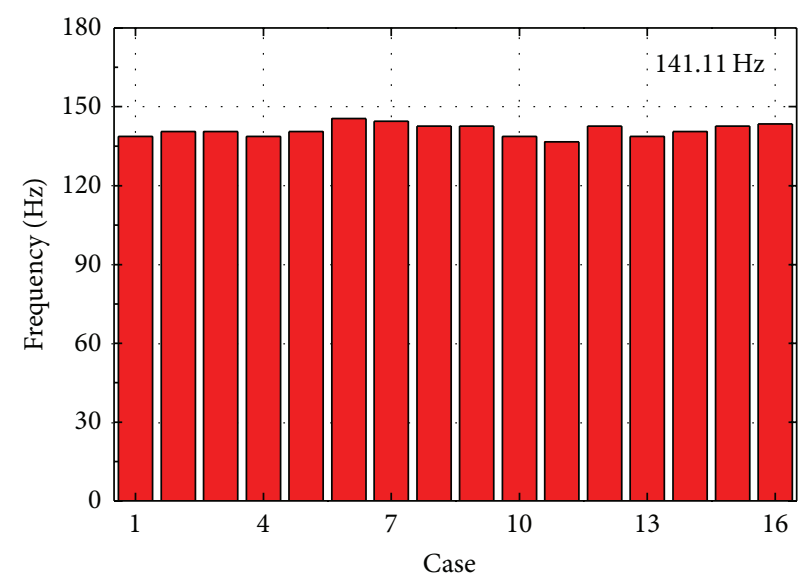

(b) Members-II

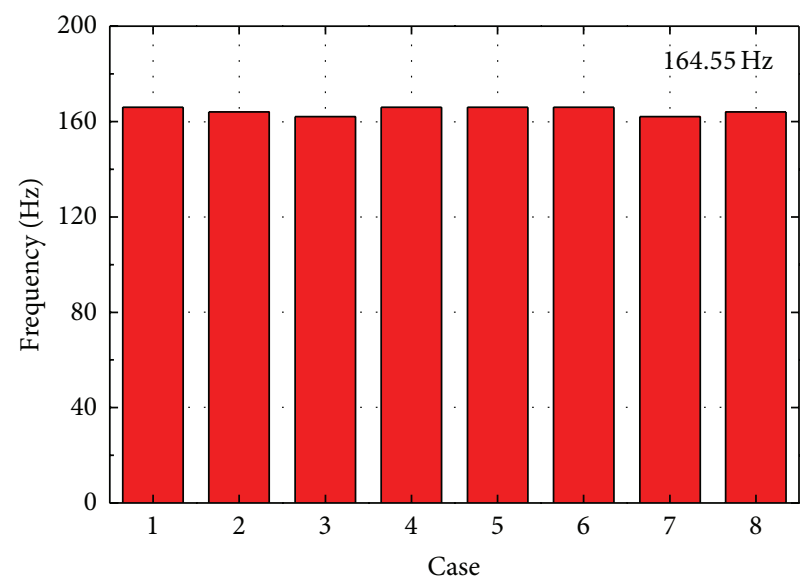

(d) Members-IV

FIgURE 11: The identified local primary frequencies.

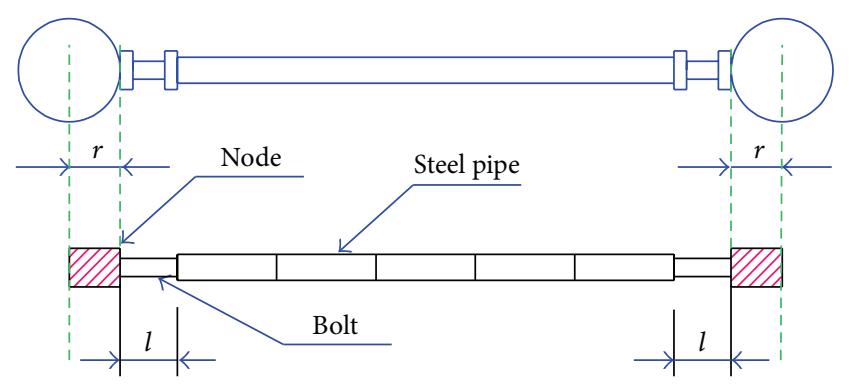

Figure 12: Fine modeling of the element.

and the definite value of $k$ needs to be optimized. In addition, the local weakening caused by bolt is considered. Denote by $l$ the weakening length, and $l$ needs to be optimized. The stiffness in the weakening range takes the value of the bolt. In addition, there are some screw holes in the spherical joint which connect with bolts and nuts, so it is hard to determine the joint weight accurately, which needs to be updated. Above all, reinforcement parameter $k$, weakening length $l$, and the joint weight $m$ are taken as the model updating parameters.

According to the model in Figure 12, a member is divided into 9 elements including 2 spherical joint elements, 2 bolt elements, and 5 pipe elements. Then the whole FE model will have 2606 nodes with 2918 elements and 15614 degrees of freedom (Dofs). Certainly the FE model is complicated and the computational work will be very huge. Therefore, model reduction is used. For each member, the fine model has 9 elements and 60 Dofs. Through Guyan reduction, the 60 Dofs are condensed into 12 Dofs on the two ends of the member, and thus the dimensions for the condensed member stiffness and mass matrix are both 12 . Then the condensed stiffness and mass matrix are used to assemble the parameter matrix of the whole FE model, except for the member with additional mass, that is, the member to be updated, of which its fine model with 60 Dofs is directly used without reduction. In this way, the consideration is balanced on the computational work, the relative accurate connection, and the accuracy of the local member analysis.

3.3.4. Model Updating. After building the fine FEM, the identified 4 modes (see Figure 7) and 58 LPFs (see Figure 11) are used to optimize the joint mass $m$, reinforcement parameter $k_{i}$, and weakening length $l_{i}$ of the four kinds of members via the objective function (6). The optimized joint mass $m$ equals $3.33 \mathrm{~kg}$, and the optimized parameters $k_{i}, l_{i}$ are shown 

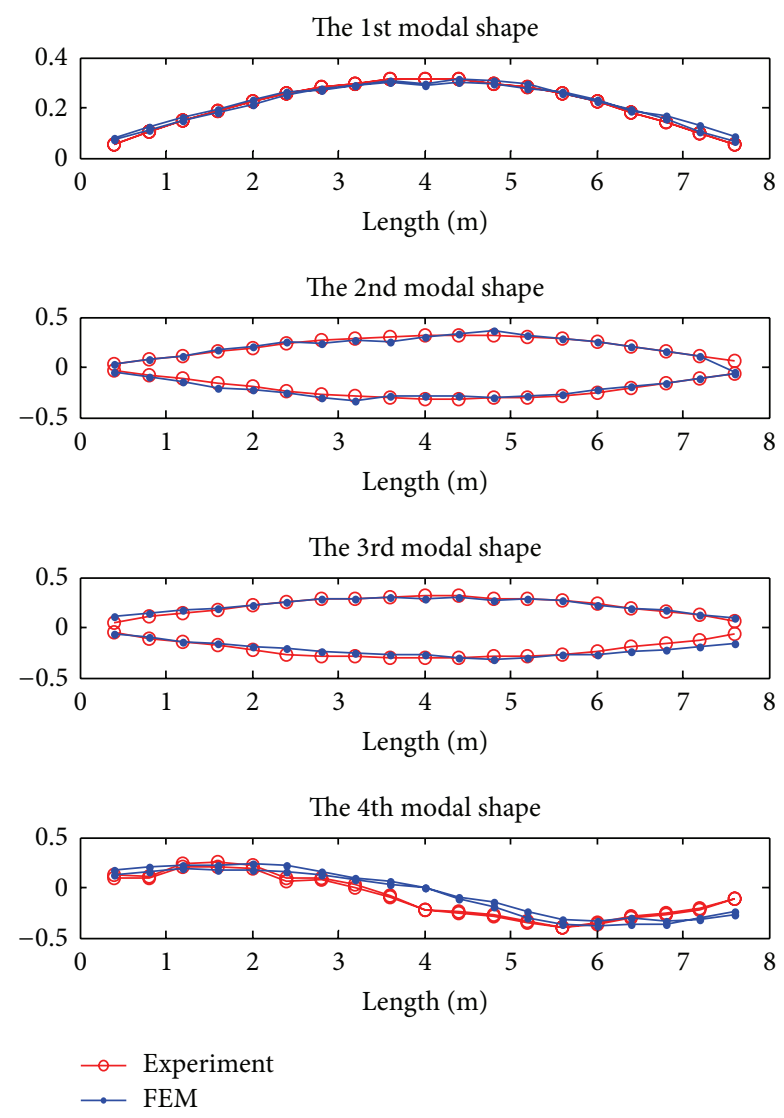

FIGURE 13: The comparison of modes of global structure.

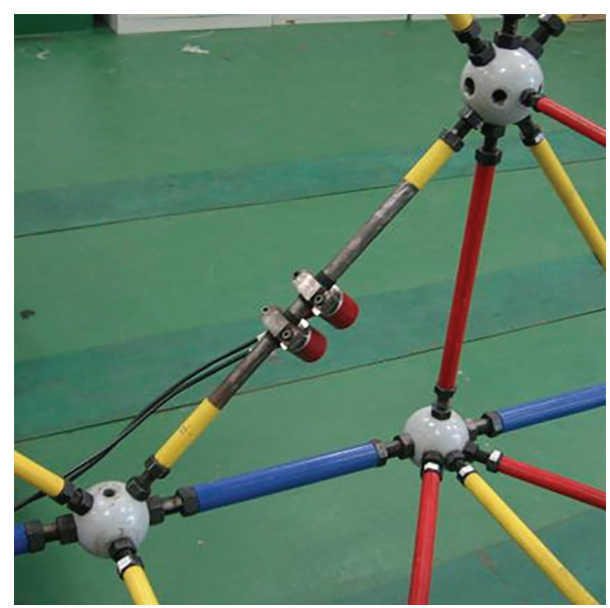

FIGURE 14: The damaged element.

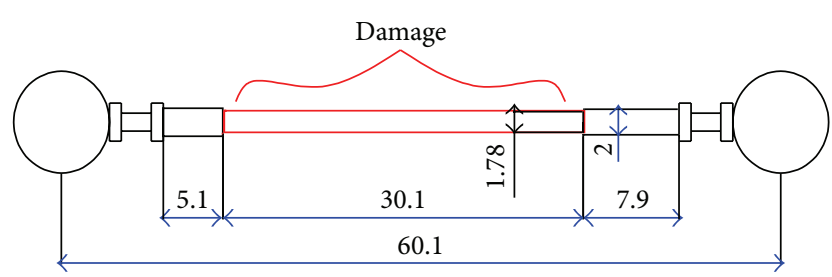

Figure 15: The actual damage of the damaged element $(\mathrm{cm})$.

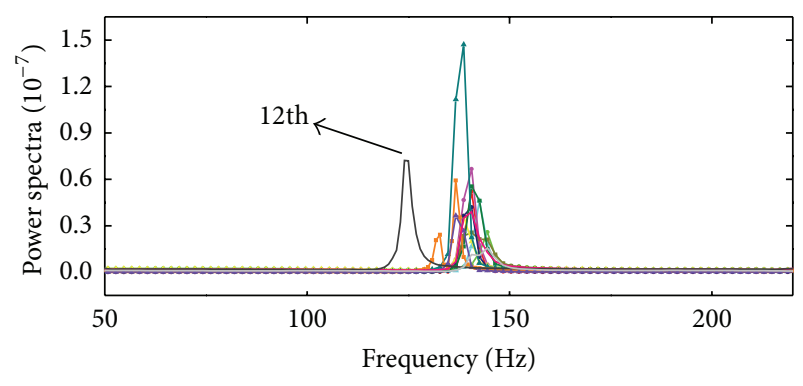

FIGURE 16: The power spetra of measured responses.

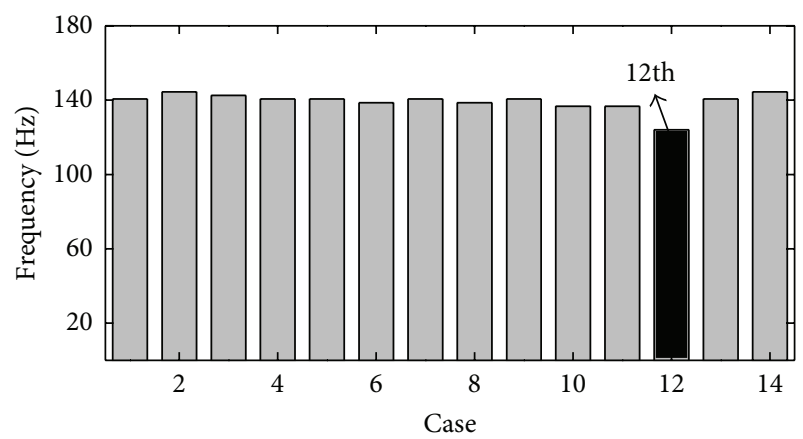

FIGURE 17: The local primary frequencies of the 14 elements.

TABLE 4: The model updating of the node parameters.

\begin{tabular}{lcc}
\hline Type & $l(\mathrm{~cm})$ & $k$ \\
\hline Members-I & 4.5 & 3.2 \\
Members-II & 2.9 & 4 \\
Members-III & 6.7 & 1.6 \\
Members-IV & 2.7 & 8 \\
\hline
\end{tabular}

TABLE 5: The errors of lower order natural frequencies of global structure.

\begin{tabular}{lccc}
\hline Order & Identified & Theoretical & Error \\
\hline 1 & 18.4 & 18.82 & $2.28 \%$ \\
2 & 27.6 & 27.25 & $-1.25 \%$ \\
3 & 36.8 & 38.63 & $4.97 \%$ \\
4 & 53.3 & 50.34 & $-5.55 \%$ \\
\hline
\end{tabular}

TABLE 6: The errors of local primary frequencies.

\begin{tabular}{lccc}
\hline Types & Identified & Estimated & Error \\
\hline Members-I & 166.02 & 162.81 & $-1.93 \%$ \\
Members-II & 140.63 & 138.99 & $-1.17 \%$ \\
Members-III & 138.67 & 138.24 & $-0.31 \%$ \\
Members-IV & 164.06 & 167.55 & $2.13 \%$ \\
\hline
\end{tabular}

in Table 4. Substitute the modified parameters into the FE model; the natural frequencies and modes of the FEM are computed and compared with the values identified from the measurements, respectively, shown in Table 5 and Figure 13. Table 6 lists some typical cases of the comparison of the LPFs between the identified values from the measurements and 
the estimated values of the updated FE model. In all the comparisons, the biggest error is $5.55 \%$, which shows that the updated FE model reflects the actual truss structure on both global dynamic behavior and local characteristic and the model is updated effectively and accurately.

3.4. Damage Identification. The type of Members-I is selected for introducing and verifying the method of damage identification using LPF. There are 14 members, and the 12th member is damaged (as shown in Figure 14). The damage dimensions of the 12th member are displayed in Figure 15, and the reduction of its flexural rigidity is $49 \%$. So the actual damage extent of the member is 0.51 , which is to be identified using LPF.

Two masses are placed in the middle of the member in the experiment; see Figure 14. The local dynamic test on Members-I, that is, 14 members, is preformed, respectively. The power spectra of the response are illustrated in Figure 16 and the identified LPFs are shown in Figure 17. As can be seen from Figure 17, 13 undamaged members show similar LPF: $140.49 \mathrm{~Hz}$ on average, while the 12 th member (the damaged one) shows slightly lower LPF: $124.02 \mathrm{~Hz}$. This not only indirectly indicates that the 12 th member is damaged, but also confirms that LPF is sensitive to the damage of the local member.

The damage extent of the 12th member is optimized using the updated FEM model. In the optimization, only one frequency (the LPF, $124.02 \mathrm{~Hz}$ ) is used for optimizing objective function (6) with the assumption that all the rest parameters are undamaged. The optimization is performed quickly and accurately. The identified damage extent of 12 th member is 0.577 , which is approximate to the actual value 0.51 .

\section{Conclusion}

This paper proposed a method for SHM using the combined structural global frequency and local frequency. An experiment of a space truss is conducted for verification of the proposed method. The obtained main conclusions are as follows.

(1) Structural global frequencies reflect the global dynamic characteristics of the structure, but they are insensitive to local modification of large structure, while structural local frequencies reflect local dynamic characteristics of the structure and they are sensitive to the variation of local parameters. So, the combination of the two kinds of frequencies can improve the accuracy of parameters estimation in SHM.

(2) In truss model, adding mass is an effective way to make the local structure have LPF, by which the local damage can be identified fast and accurately. The relation analysis among the adding masses, LPF, and the relative sensitivity is discussed as the evidence to obtain the LPF. However, when large value of additional mass is required, some challenges are encountered in actual operation and mass installation, which are subjects of an ongoing research.

\section{Conflict of Interests}

The authors declare that there is no conflict of interests regarding the publication of this paper.

\section{Acknowledgments}

The authors gratefully acknowledge the support of the Major State Basic Research Development Program of China (973 Program) (2013CB036305), of National Science Foundation of China (NSFC) (51108057, 51108066), of the Fundamental Research Funds for the Central Universities (China) (DUT13LK13), of Special Financial Grant from the China Postdoctoral Science Foundation (2012T50255), and of the Project of National Key Technology R\&D Program (China) (2011BAK02B01, 2011BAK02B03, and 2006BAJ03B05). Financial support of the Polish National Science Centre Project "AIA" (DEC-2012/05/B/ST8/02971) and of Structural Funds in the Operational ProgrammeInnovative Economy (IE OP) financed from the European Regional Development Fund, Project "Modern material technologies in aerospace industry" (POIG.01.01.02-00015/08), is gratefully acknowledged.

\section{References}

[1] P. C. Chang, A. Flatau, and S. C. Liu, "Review paper: health monitoring of civil infrastructure," Structural Health Monitoring, vol. 2, no. 3, pp. 257-267, 2003.

[2] E. Figueiredo, G. Park, C. R. Farrar, K. Worden, and J. Figueiras, "Machine learning algorithms for damage detection under operational and environmental variability," Structural Health Monitoring, vol. 10, no. 6, pp. 559-572, 2011.

[3] W. Fan and P. Qiao, "Vibration-based damage identification methods: a review and comparative study," Structural Health Monitoring, vol. 10, no. 1, pp. 83-111, 2011.

[4] S. Hassiotis, "Identification of damage using natural frequencies and Markov parameters," Computers and Structures, vol. 74, no. 3, pp. 365-373, 2000.

[5] Z. Duan, G. Yan, J. Ou, and B. F. Spencer, "Damage localization in ambient vibration by constructing proportional flexibility matrix," Journal of Sound and Vibration, vol. 284, no. 1-2, pp. 455-466, 2005.

[6] R. M. Lin and D. J. Ewins, "Model updating using FRF data," in Proceedings of the 15th International Seminar on Modal Analysis, pp. 141-162, Leuven, Belgium, 1990.

[7] C.-B. Yun and E. Y. Bahng, "Substructural identification using neural networks," Computers and Structures, vol. 77, no. 1, pp. 41-52, 2000.

[8] B. Jaishi and W.-X. Ren, "Finite element model updating based on eigenvalue and strain energy residuals using multiobjective optimisation technique," Mechanical Systems and Signal Processing, vol. 21, no. 5, pp. 2295-2317, 2007.

[9] Y. An and J. Ou, "Experimental and numerical studies on model updating method of damage severity identification utilizing four cost functions," Structural Control and Health Monitoring, vol. 2o, no. 1, pp. 107-120, 2013.

[10] N. G. Nalitolela, J. E. T. Penny, and M. I. Friswell, "Mass or stiffness addition technique for structural parameter updating," The International Journal of Analytical and Experimental Modal Analysis, vol. 7, no. 3, pp. 157-168, 1992. 
[11] N. Nalitolela, J. E. T. Penny, and M. I. Friswell, "Updating model parameters by adding an imagined stiffness to the structure," Mechanical Systems and Signal Processing, vol. 7, no. 2, pp. 161172, 1993.

[12] P. D. Cha and L. G. de Pillis, "Model updating by adding known masses," International Journal for Numerical Methods in Engineering, vol. 50, no. 11, pp. 2547-2571, 2001.

[13] H. M. Dinh, T. Nagayama, and Y. Fujino, "Structural parameter identification by use of additional known masses and its experimental application," Structural Control and Health Monitoring, vol. 19, no. 3, pp. 436-450, 2012.

[14] J. L. Hou, L. Jankowski, and J. P. Ou, "Substructural damage identification using local primary frequency," in Proceedings of the 11th International Symposium on Structural Engineering, Guangzhou, China, December 2010.

[15] J. L. Hou, L. Jankowski, and J. P. Ou, "Structural damage identification by adding virtual masses," Structural and Multidisciplinary Optimization, vol. 48, pp. 59-72, 2013. 


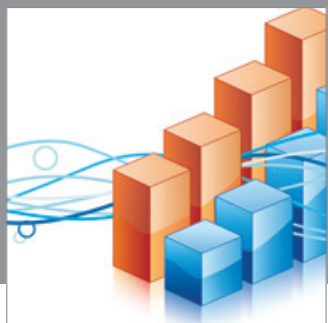

Advances in

Operations Research

mansans

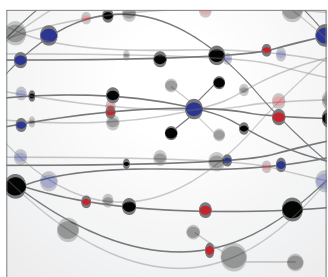

The Scientific World Journal
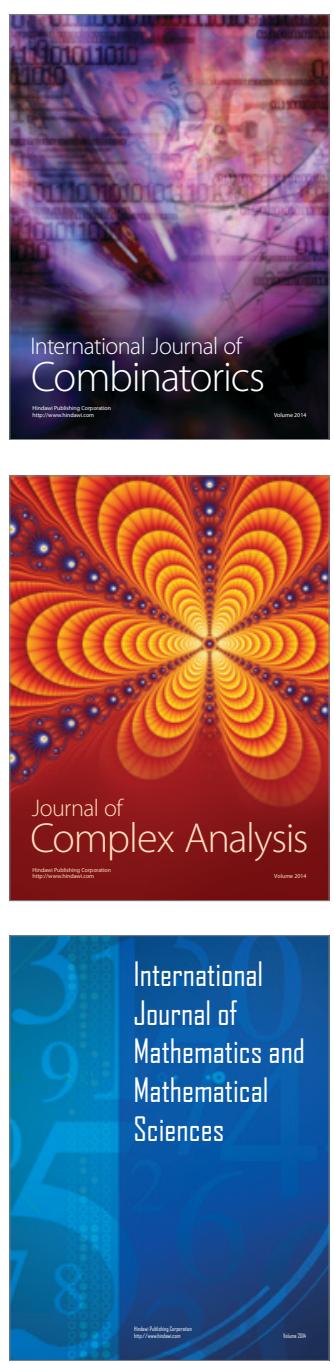
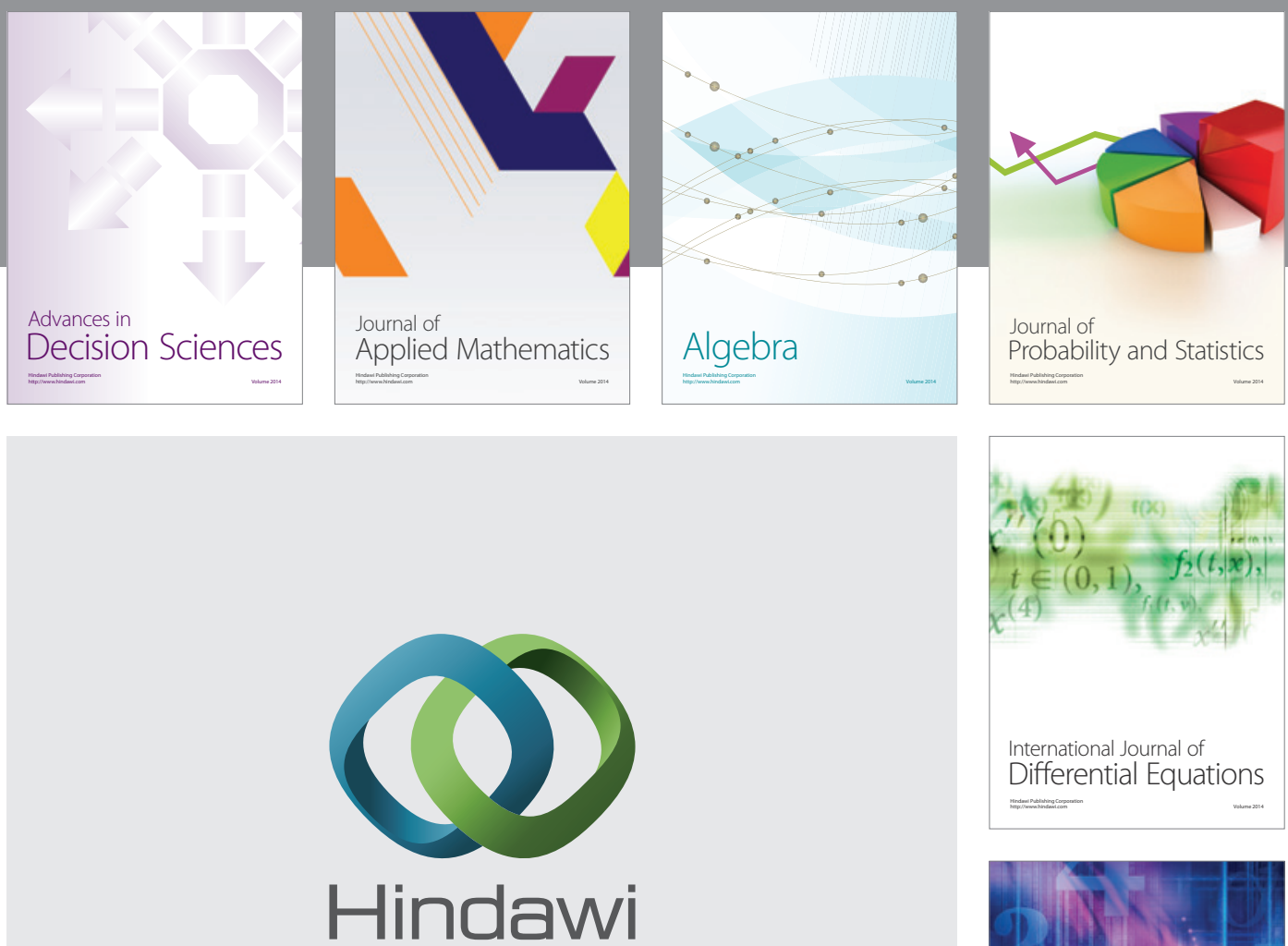

Submit your manuscripts at http://www.hindawi.com
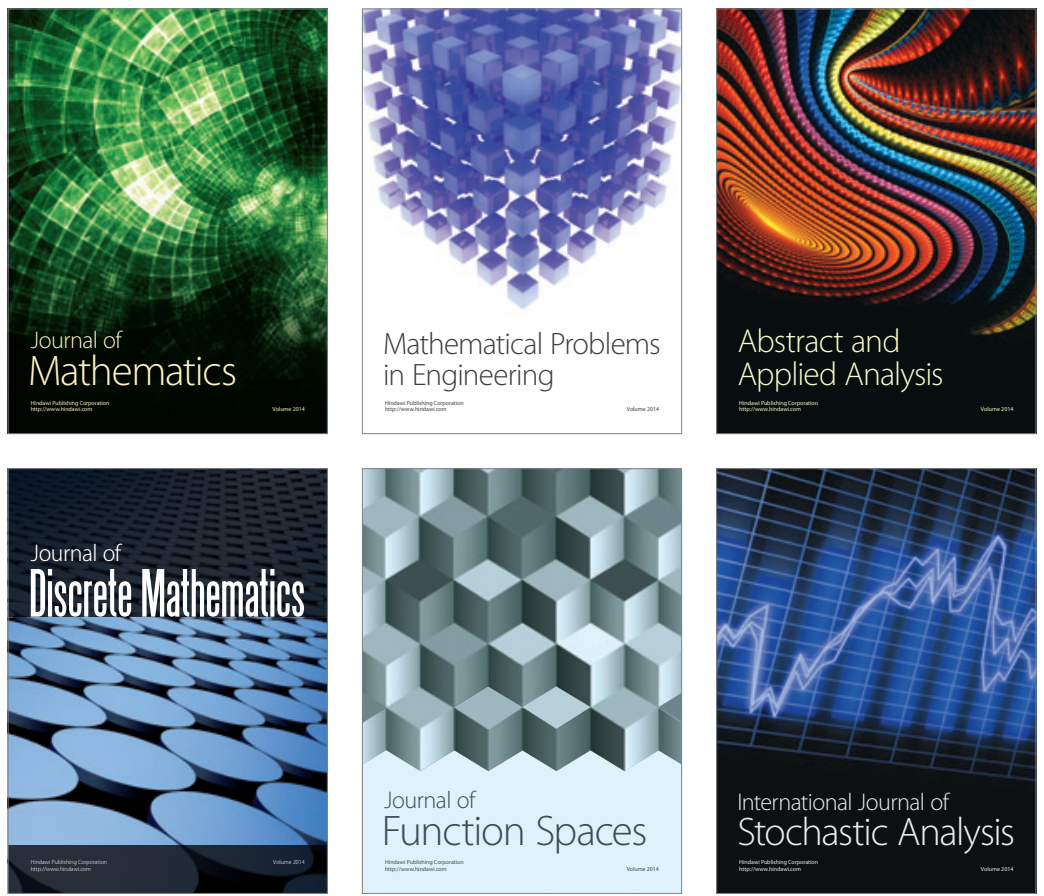

Journal of

Function Spaces

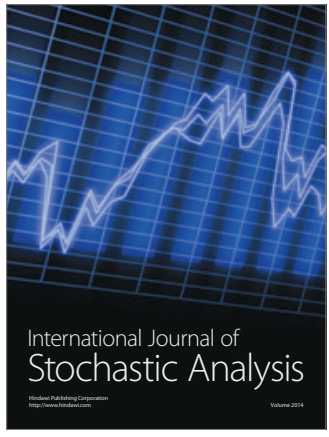

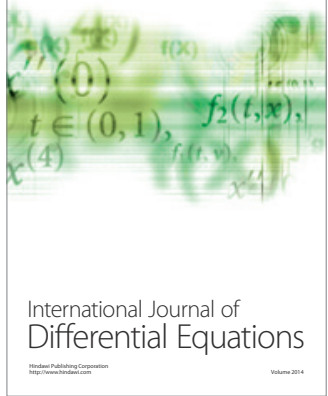
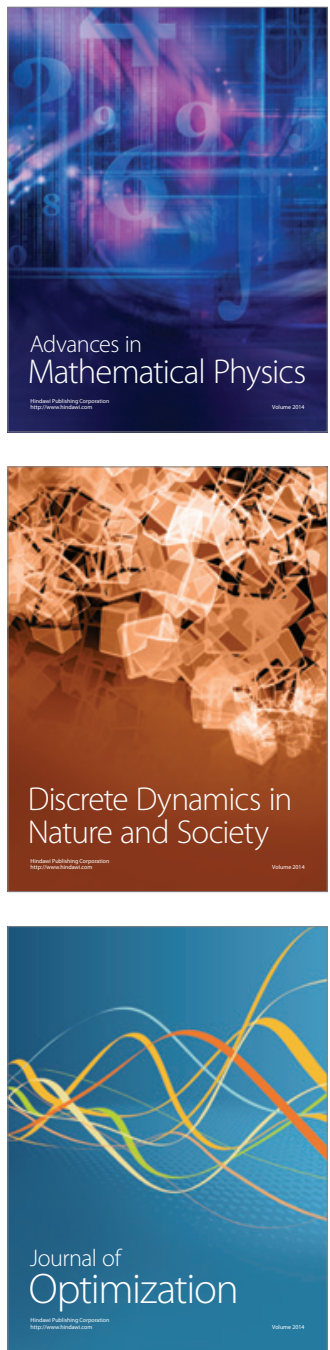